\title{
Electron optics of microlenses with inclined beams
}

\author{
Y. Zhang, ${ }^{\text {a) }}$ J. E. Barth, and P. Kruit \\ Faculty of Applied Sciences, Delft University of Technology, Lorentzweg 1, 2628 CJ Delft, the Netherlands
}

(Received 4 January 2008; accepted 20 February 2008; published 1 April 2008)

\begin{abstract}
For multielectron beam systems with a single electron source, the outside beams need to be collimated before entering the individual microcolumns. As an alternative of the traditional multibeam source design where the broad beam from the source is collimated by a single lens, the broad beam can be first split in subbeams that are focused by a microlens array and subsequently collimated by a deflector array. This configuration avoids the large angular error due to spherical and chromatic aberration of the collimator lens. The electron optics of the microlenses that are focusing skewed beams is investigated for different lens types and geometries. A two-electrode immersion microlens array with offset apertures is a good candidate due to its small off-axial aberrations. A 100-beam source unit, comprising a Schottky emitter, an immersion microlens array, and a deflector array, is capable of generating 100 parallel beamlets with beamlet current of $2 \mathrm{nA}$.
\end{abstract}

(C) 2008 American Vacuum Society. [DOI: 10.1116/1.2897321]

\section{INTRODUCTION}

The technology of microelectromechanical systems offers the possibility of making microsized electrostatic lenses, deflectors, blankers, and multipoles. ${ }^{1}$ A single-beam miniature electron column is capable of writing sub- 100 -nm patterns and resolving less than $35 \mathrm{~nm}$ features. ${ }^{2}$ By arraying such miniature columns ${ }^{3-7}$ or stacking microsized optical component arrays ${ }^{8-12}$ to create plural focused and individually controlled beams, the total current delivered to the wafers can be increased without suffering from source brightness limitation and Coulomb interactions. Multibeam systems with stacked optical component arrays are good candidates for maskless lithography where thousands of beams are necessary to meet the throughput requirements.

In multibeam systems with stacked optical arrays, the broad beam is usually collimated, subsequently split into multiple beams by an aperture array, focused by a microlens array, and controlled via a blanker array located at the conjugate plane. For high resolution applications, the size of the lens array or the throughput is limited by the off-axial aberrations of the collimator lens. ${ }^{13}$ Alternatively, the broad beam may impinge on an aperture array and lens array obliquely, and the beamlets are collimated by a deflector array at the conjugate plane. This configuration provides a solution of avoiding the angular error due to spherical and chromatic aberrations of a collimator lens: ${ }^{14}$ by focusing each beamlet at the deflector array, the opening angle in the beam can be made substantially larger than the chromatic angular error; and the spherical angular error in the collimator lens is eliminated by collimating using the deflector array.

The off-axial aberrations of the lens array with skewed incidence can be minimized through the use of offset current limiting apertures. This is first proposed by Kurihara ${ }^{15}$ for an Einzel lens matrix with skewed incidence, where field curvature is corrected by changing the focus using a correction lens array. However, the beamlet incident angle is restricted

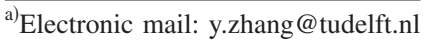

to $30 \mathrm{mrad}$ due to astigmatism and the throughput is limited by the number of beamlets. The alignment between the Einzel lens array, aperture array, and the correction lens array raises serious engineering challenges.

In this work, the electron optics of microlenses with skewed incidence is investigated for different lens types and geometries. It shows that a two-electrode immersion microlens array is a good candidate for generating multiple probes with small off-axial aberrations. A multibeam source is designed, where the aperture array is integrated with the microlens array and the field curvature is corrected by adjusting the diameters of the outer lenses. The performance of the multibeam source is evaluated.

\section{METHOD OF ANALYSIS}

The typical dimensions of microlenses are around $50-150 \mu \mathrm{m}$ for a beam voltage of $1-5 \mathrm{kV}$. For a $10 \times 10$ microlens array with skewed incidence, the off-axial distance for the outermost lens is more than $0.25 \mathrm{~mm}$ and the offaxial aberrations deteriorate the images of outer lenses. The off-axial aberrations, however, can be reduced by offsetting the current limiting aperture from the axis [Fig. 1(a)] such that the "virtual aperture" is centered in the lens plane [Fig. 1(b)]. For electrostatic lenses where a lens-plane aperture interrupts the lens field, the aperture should be in a field-free region.

The off-axial aberrations can be evaluated by deviations from the paraxial trajectory in the Gaussian image plane. The third order radial deviations can be calculated using formulas in Table I, where $S_{A}, K_{A}, F_{A}, A_{A}, D_{A}, X_{A}^{*}$, and $T_{A}^{*}$ are aperture-dependent aberration coefficients, and $\alpha_{a}$ and $\beta_{o}$ are the complex slope and complex coordinate in the object plane. ${ }^{16,17}$ The aberration coefficients are obtained from a two-dimensional finite element method programelectrostatic lens design (ELD). ${ }^{18}$ The radius of the total blur is roughly estimated by the square root square sum of the deviations. 

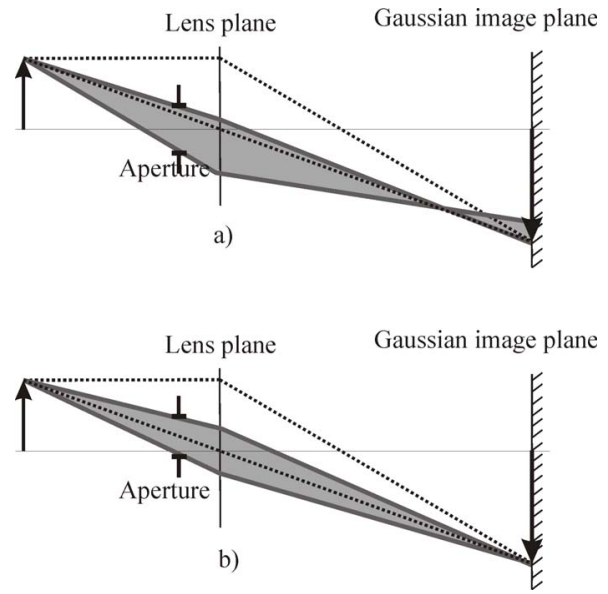

FIG. 1. Off-axial aberrations with (a) an aperture on the axis and (b) an offset aperture such that the virtual aperture is centered in the lens plane.

The plane of best focus, in general, does not coincide exactly with the Gaussian image plane. By taking into account only the spherical aberration, for example, the disk of least confusion is only one-fourth as large as the circle of confusion in the Gaussian image plane. In the world of analytical microscopy and lithography, people use the FW50 disk (the minimum spot diameter containing 50\% probe current) for probe size characterization: the spherical FW50 disk is only $9 \%$ of the circle of confusion in the Gaussian image plane. ${ }^{19}$ As the microlens array may be used for applications in lithography or inspection, it is important to evaluate the FW50 blur. A finite difference method program, SIMION, is used for this purpose: 500 electrons emitted from an infinitely small spot with pseudorandom angular distribution and zero energy spread are traced through the microlenses; positions and angles of each electron are recorded and input into MATLAB, where through focus series are produced and the FW50 blur is determined.

\section{ABERRATION-MINIMIZED MICROLENS ARRAY}

The aberrations for an electrostatic lens are dependent on lens type, geometry, and the aperture position. Computer models of an Einzel lens array and a two-electrode immersion lens array are compared at the image side, with the same potential, focal length, magnification, and half opening angle. The immersion lens or Einzel lens is composed of two/three $400 \mu \mathrm{m}$ thick electrodes with a $400 \mu \mathrm{m}$ separa-

TABLE I. Radial deviations from the paraxial trajectory in the Gaussian image plane for optical systems with apertures.

\begin{tabular}{lll}
\hline \hline Geometric aberrations & Spherical & $M S_{A} \alpha_{a}^{2} \bar{\alpha}_{a}$ \\
& Coma & $M K_{A} \alpha_{a} \beta_{o} \bar{\alpha}_{a}$ \\
& Field curvature & $M F_{A} \alpha_{a} \beta_{o} \bar{\beta}_{o}$ \\
& Astigmatism & $M A_{A} \beta_{o}^{2} \bar{\alpha}_{a}$ \\
& Distortion & $M D_{A} \beta_{o}^{2} \bar{\beta}_{o}$ \\
Chromatic aberration & Axial & $M X_{A}^{*} \alpha_{a} \Delta V / V$ \\
& Transverse & $M T_{A}^{*} \beta_{o} \Delta V / V$ \\
\hline
\end{tabular}

TABLE II. Optical properties of the axial Einzel lens and the two-electrode immersion lens.

\begin{tabular}{lcc}
\hline \hline & & $\begin{array}{c}\text { two-electrode immersion } \\
\text { lens }\end{array}$ \\
\hline Potential at object side (V) & 8000 & 4000 \\
Potential at image side (V) & 8000 & 8000 \\
Potential at middle electrode (V) & 5067 & N.A. \\
Focal length at image side (mm) & 8.72 & 8.72 \\
Magnification & -5.0 & -5.0 \\
Angular magnification & -0.20 & -0.14 \\
Half-opening angle image side & 0.38 & 0.38 \\
$\quad$ (mrad) & & \\
Spherical FW50 (nm) & 65.0 & 71.5 \\
Chromatic FW50 (nm) & 6.3 & 8.4 \\
\hline
\end{tabular}

tion, and the lens radii are $200 \mu \mathrm{m}$. The potentials of the electrodes are determined such that both lenses have the same focal length at the image side. The object plane is chosen to have the same magnification. The half-opening angle is chosen such that the beam is around $10 \%$ of the lens diameter, which is usually the maximum filling for conventional lenses. The optical properties for the axial lenses are listed in Table II. Note that the spherical and chromatic FW50 disks are different from their deviations in the Gaussian image plane.

The aperture-dependent aberration coefficients of the lenses are obtained in ELD. The deviations in the Gaussian image plane are calculated using formula in Table I for an outer lens with an off-axial distance of $1 \mathrm{~mm}$ (equal to an incident angle of $115 \mathrm{mrad})$. The deviations are plotted in Fig. 2 as a function of the $z$ position of the virtual aperture, with the origin located at the geometrical center of the lenses. The axial blur is indicated by a star on the $y$ axis. The total blur $(\mathrm{C}+\mathrm{A}+\mathrm{S}+\mathrm{Chro})$ is estimated as square root square sum of coma, astigmatism, spherical, and chromatic aberration. Field curvature can be compensated by adjusting the lens diameters as will be explained in the next paragraph. Even though having similar axial aberrations, it is clear in Fig. 2 that the off-axial aberrations of the immersion lens are much smaller than that of the Einzel lens: the total blur is $1268 \mathrm{~nm}$ for the outer Einzel lens and $506 \mathrm{~nm}$ for the outer immersion lens. For the immersion lens, a $50 \mu \mathrm{m}$ shift from the optimum aperture position, which, with an incident angle of $115 \mathrm{mrad}$ corresponds to a misalignment of $5.75 \mu \mathrm{m}$ between the aperture and lens, gives a rise to about $25 \mathrm{~nm}$ in the total blur.

The immersion microlenses can be modeled by two coaxial cylinders with equal diameters and separated by a small distance. Numerical calculations show that for a given ratio of electrode potentials, the focal length becomes larger by increasing the lens diameter. ${ }^{20}$ Therefore, it is possible to correct field curvature by enlarging the diameter of the outer lenses. With offset apertures and the field curvature correction technique, a large-sized multibeam system may be made with the immersion microlens array. 


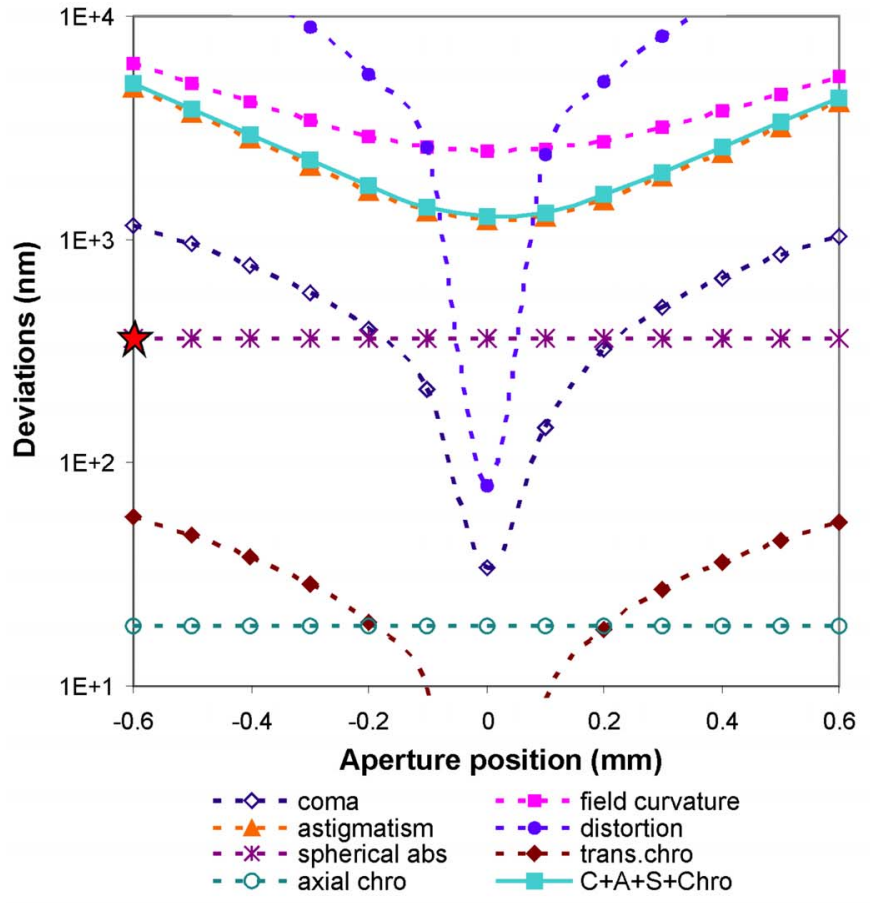

a)

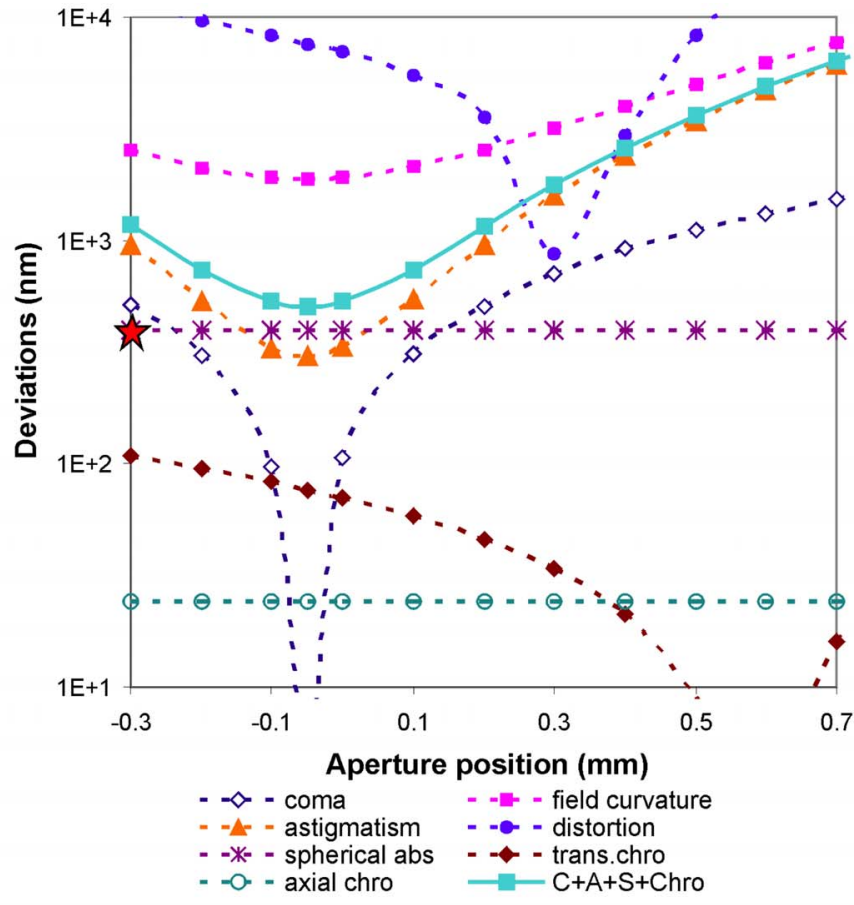

b)

Fig. 2. Deviations and total blur in the Gaussian image plane for (a) an outer Einzel lens at an off-axial distance of $1 \mathrm{~mm}$ and (b) an outer two-electrode immersion lens at an off-axial distance of $1 \mathrm{~mm}$.

\section{MICROLENS ARRAY IN MULTIBEAM SYSTEMS}

The microlens array with skewed incidence may be used in multibeam systems to improve the throughput by deliver-

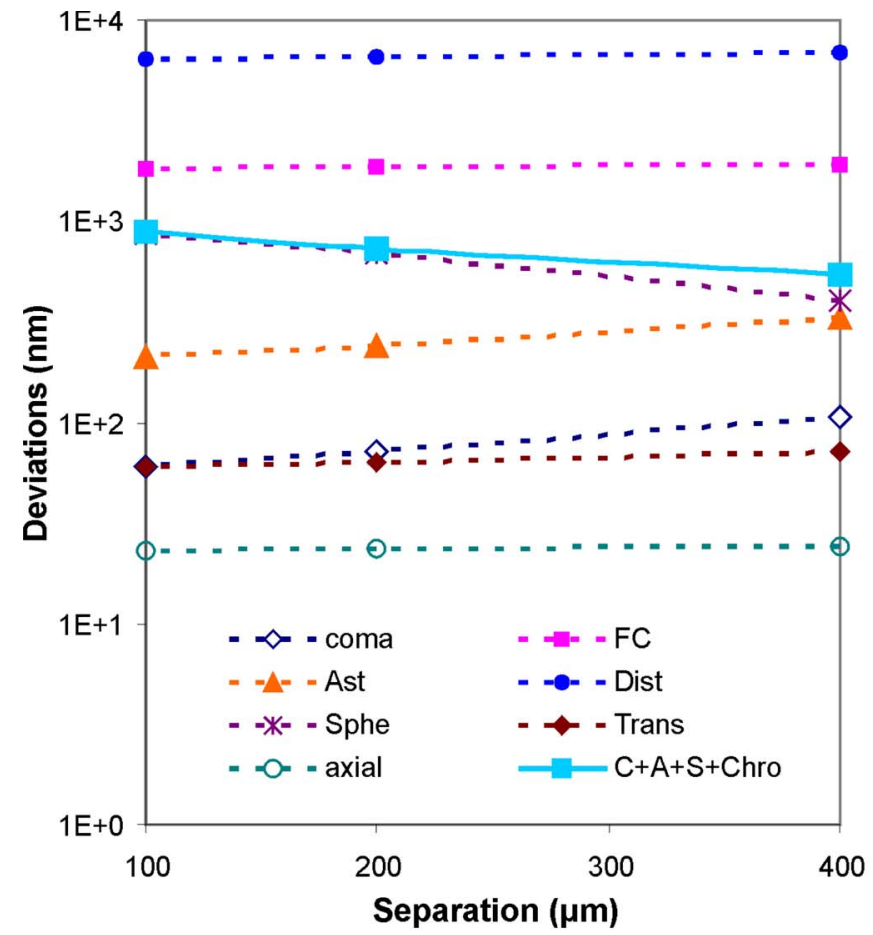

a) ing more current than a single-beam system. The total current transmitted through the microlens array can be expressed as

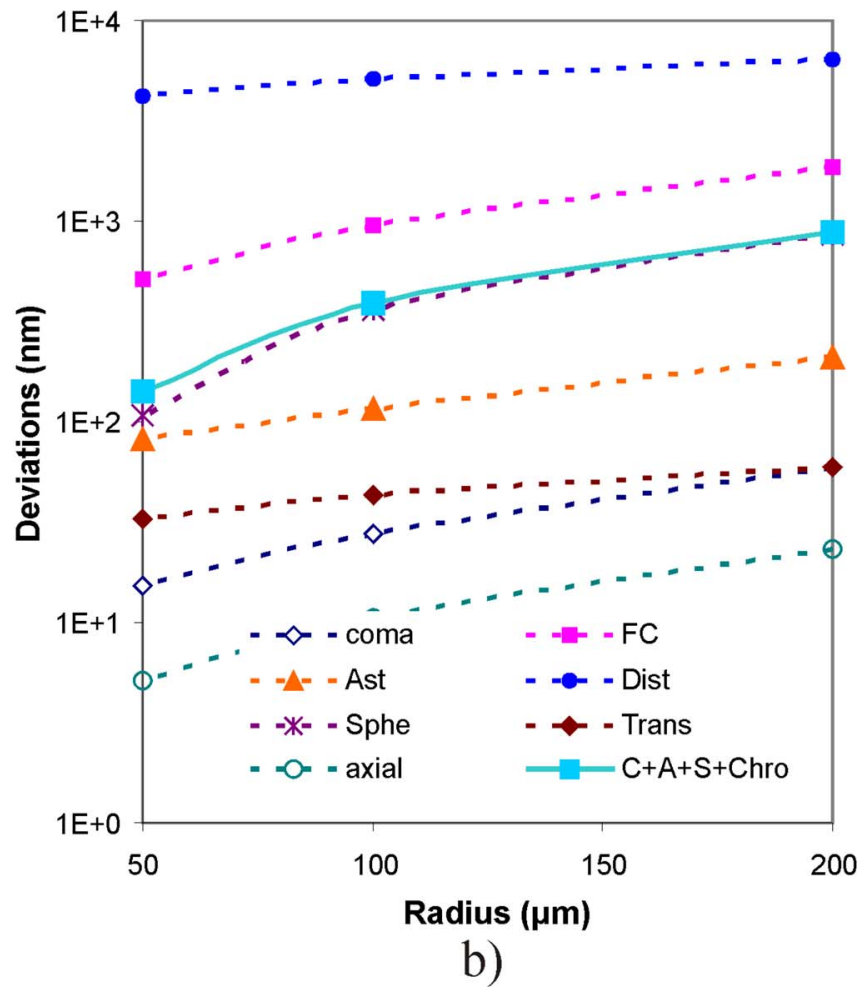

Fig. 3. Relationship of the lens geometry and deviations of the outermost lens in the microlens array, when keeping the focal length, magnification, filling factor, and the total current the same: (a) deviations as a function of lens electrode separation (lens radius equals to $200 \mu \mathrm{m}$ ); (b) deviations as a function of lens radius (lens separation equals to $100 \mu \mathrm{m}$ ). 


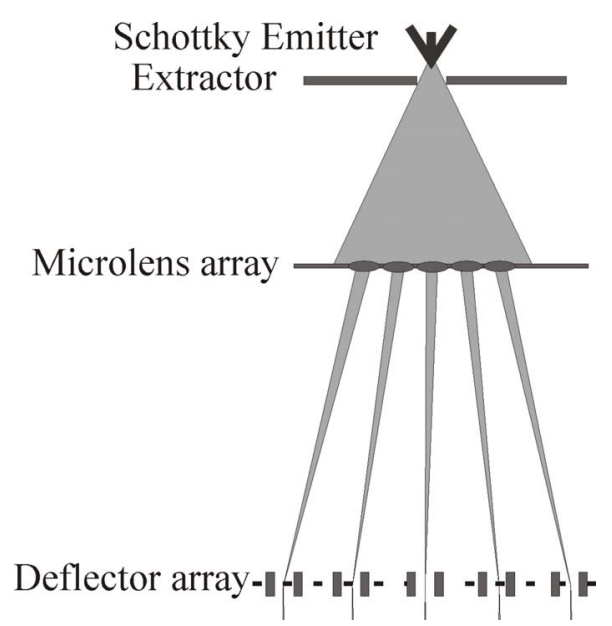

FIG. 4. Configuration of the multibeam source.

$$
I_{\text {total }} \propto j \pi \alpha^{2}\left(\frac{d_{\text {lens }}}{P_{\text {lens }}}\right)^{2} \mathrm{FF}^{2},
$$

where $\alpha$ is the electron source half-emission angle, within which the angular current density $j$ is uniform. These two parameters are determined by the type of electron sources and the source operation conditions. High brightness Schottky sources are preferred in lithography and microscopy system which demand both high resolution and high current density. Schottky sources and their operation conditions will not be discussed in this article, but more details can be found in Ref. $21 d_{\text {lens }} / P_{\text {lens }}$ is the ratio of lens diameter and lens pitch, which is limited around 0.8 to maintain enough physical strength of the lens electrodes and prevent electrostatic bending FF is the filling factor defined as the ratio of beam diameter in the lens plane and the lens diameter. The maximal filling factor of a conventional lens is around $10 \%$ and obtained when the FW50 blur (due to the

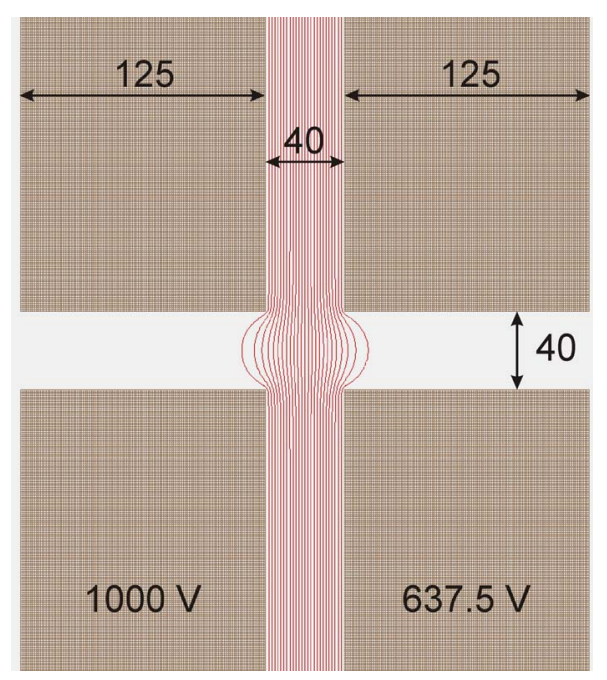

FIG. 5. Geometry (unit: micron), potential and equipotential lines of the two-electrode immersion lens with integrated current limiting aperture in a field-free region.
TABLE III. Image side optical properties of the axial microlens.

\begin{tabular}{ll}
\hline \hline Objective distance & $2.7 \mathrm{~mm}$ \\
Image distance & $4.8 \mathrm{~mm}$ \\
Magnification & -2.0 \\
Angular magnification & -0.63 \\
Focal length & $1.55 \mathrm{~mm}$ \\
Geometrical spot size & $100 \mathrm{~nm}$ \\
Spherical coefficient & $1.74 \times 10^{5} \mathrm{~mm}$ \\
Axial chromatic coefficient & $2.1 \times 10^{1} \mathrm{~mm}$ \\
Half opening angle & $1.16 \mathrm{mrad}$ \\
Spherical FW50 & $49.1 \mathrm{~nm}$ \\
Chromatic FW50 & $6.7 \mathrm{~nm}$ \\
Filling factor & $25 \%$ \\
\hline \hline
\end{tabular}

Coulomb interactions and aberrations) reaches the geometrical spot size. In multibeam systems, effects of the Coulomb interactions are greatly reduced by splitting the broad beam into beamlets. In order to have a large total current, the aberrations of the microlenses should be minimized to allow for a large filling factor.

The aberrations of the microlenses are investigated for different lens geometries. The comparison is carried out under the condition that the total current transmitted through the microlens arrays is equal. Assuming identical source parameters (angular current density and useful source halfemission angle) and $d_{\text {lens }} / P_{\text {lens }}$, the filling factor should be the same. The outermost lenses in the microlens arrays, for which the incident angles are $115 \mathrm{mrad}$, are compared at the same focal length, magnification, and beam potential at the image side.

First, the deviations in the Gaussian image plane are plotted in Fig. 3(a) for different electrode separations, while the lens radius is fixed at $200 \mu \mathrm{m}$. The total deviation increases from 536 to $894 \mathrm{~nm}$ by changing the electrode separation from 400 to $100 \mu \mathrm{m}$, with decreasing astigmatism and coma, and increasing spherical deviation. The total blur, when keeping the separation at $100 \mu \mathrm{m}$, however, decreases

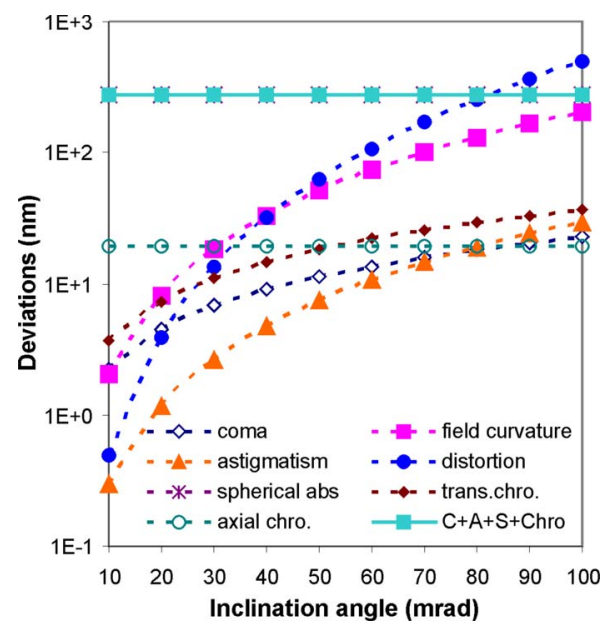

FIG. 6. Deviations and total blur as a function of incident angle on the microlens array. 

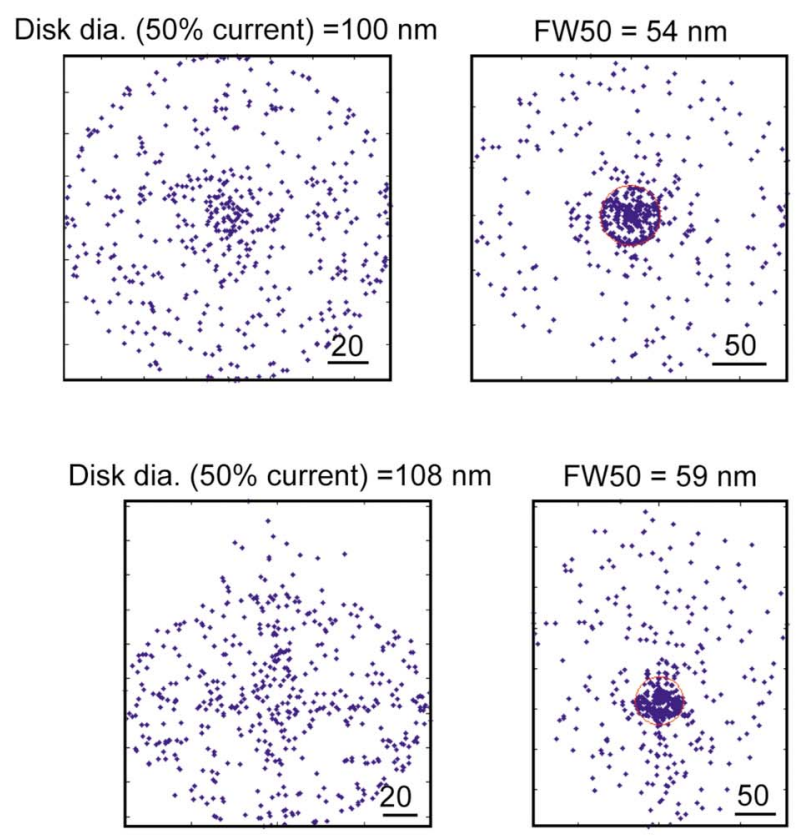

$\mathrm{FW} 50=59 \mathrm{~nm}$

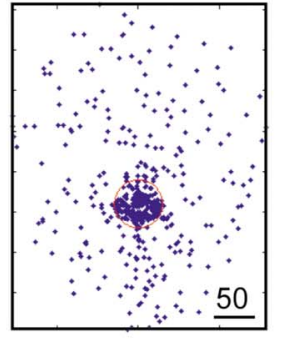

Disk dia. $(50 \%$ current $)=216 \mathrm{~nm}$

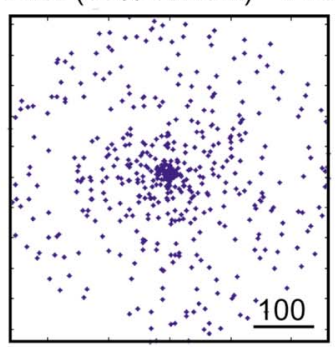

Disk dia. $(50 \%$ current $)=228 \mathrm{~nm}$

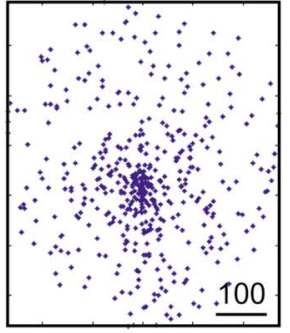

FIG. 7. Through focus series of the microlenses (a) axial lens and (b) an outer lens with inclination angle of $90 \mathrm{mrad}$. The FW50 blur is indicated by a circle. to $141 \mathrm{~nm}$ by choosing a lens radius of $50 \mu \mathrm{m}$, as shown in Fig. 3(b). This seems counterintuitive since the lens aberrations of each lens increase with decreasing radius, but the reader is reminded here that the filling factor is kept constant and that the total number of lenses is increased. A smaller total blur then allows a larger filling factor (or an opening angle) until the FW50 blur reaches about half the geometrical spot size. The advantages are a larger total current and a spherical aberration-dominated blur, which is preferred in terms of spot size uniformity. (Spherical aberration is proportional to the cube of the opening angle and increases faster than other aberrations.)

For a microlens array to be used in multibeam systems, the lens pitch is tens of microns in order to put the probes at a typical pitch of $150 \mu \mathrm{m}$. The current per beamlet is around several nanoamperes. The electrode separation is a trade-off between the spherical aberration, astigmatism, and practical implementation. As an example, one particular multibeam source system is designed and the performance is evaluated.

\section{A. The configuration of the multibeam source}

The multibeam source is designed to generate 100 parallel beamlets and the configuration is shown in Fig. 4. The broad beam emitted from the Schottky emitter is split by an aperture array and beamlets are projected by the microlens array at the center of the deflector electrodes. Subsequently, the beamlets are collimated by applying a voltage proportional to the landing angles at the deflector array. To reduce the deflection voltage for the outer beams, the extraction voltage is set at $1 \mathrm{kV}$. The distance between the tip and the extractor is adjusted to have sufficient field near the tip. Furthermore, a deceleration microlens is used to restrict the deflection voltage below $15 \mathrm{~V}$. The 100-beam source unit should be able to work either as a single unit or arranged in an array to generate massive parallel beamlets for maskless lithography. ${ }^{22,23}$

The distance between the tip and the aperture array, where most of the trajectory displacement occurs, is set to $2.7 \mathrm{~mm}$. The microlenses are arranged hexagonally in a pitch of $50 \mu \mathrm{m}$. The lens configuration and equipotential lines are illustrated in Fig. 5. As shown, the lens field is locally limited in the bore, thus the current limiting aperture can be integrated on the first electrode and sit in a field-free region. The integration improves the alignment between the lens array and aperture array by aligning with a mask aligner, which has typical submicron alignment accuracy.

\section{B. Multibeam source performance}

The optical properties of the axial microlens are listed in Table III. The microlens array projects multiple source images in a pitch of around $140 \mu \mathrm{m}$. The filling factor is limited to $25 \%$ by the spherical FW50 disk. Assuming the lowextraction-voltage Schottky emitter having a brightness of $10^{8} \mathrm{~A} / \mathrm{m}^{2} \mathrm{sr} \mathrm{V}$, a virtual source size of $50 \mathrm{~nm}$ and an angular current density of $200 \mu \mathrm{A} / \mathrm{sr}$, then the current per beamlet is $2.15 \mathrm{nA}$, and the total current at wafer is $215 \mathrm{nA}$.

The deviations in the Gaussian image plane are plotted in Fig. 6, as a function of the incident angle on the microlens array. The total blur is dominated by the spherical aberration.

The geometrical FW50 blur (including spherical aberration, coma, and astigmatism) for the outer lenses are determined more precisely using through focus series. The through focus series (100 $\mu \mathrm{m}$ before and after the best focus plane) for the central lens is shown in Fig. 7(a). The beam profile changes rapidly with image positions and only spherical aberration is present in the probe. The FW50 blur is $54 \mathrm{~nm}$, indicated with a circle in the second image. The 


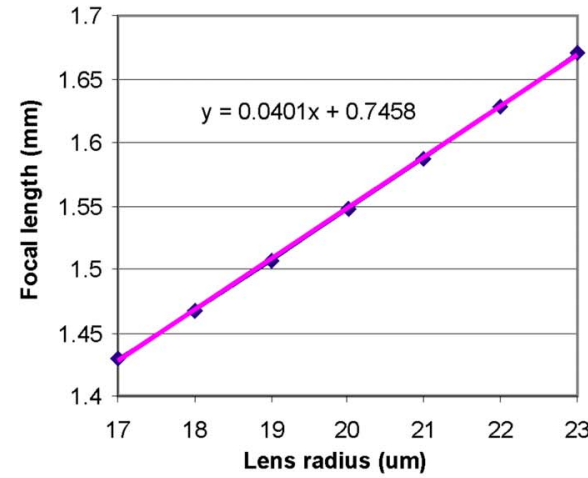

a)

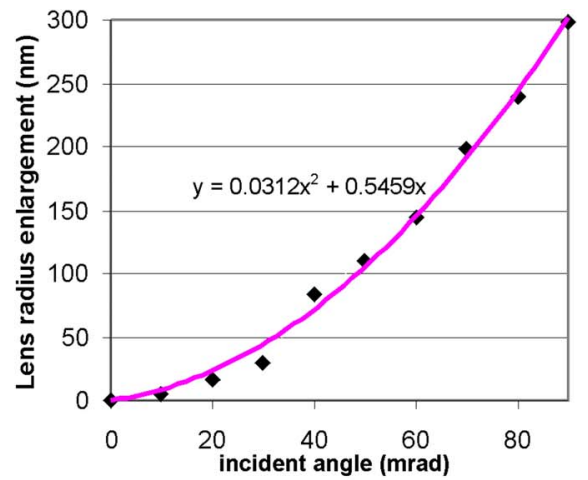

b)
FIG. 8. Relationship of the focal length and lens radius and (b) the radii enlargement for outer lenses for full compensation of field curvature. slight difference from the theoretic value in Table III may be due to higher order aberrations in the through focus series. The geometrical FW50 blur for an outer lens with incident angle of $90 \mathrm{mrad}$ is shown in Fig. 7(b), where coma and astigmatism are present besides the spherical aberration. The FW50 blur rises to $59 \mathrm{~nm}$.

The total spot size can be obtained using the power root sum (RPS) algorithm, ${ }^{19}$ by adding the spherical aberration dominated FW50 blur $\left(d_{\mathrm{FWs} 50}\right)$ and the geometrical spot size $\left(d_{\text {geo }}\right)$ using power $=1.3$; the result is then added with the chromatic FW50 blur $\left(d_{\mathrm{FWc50}}\right)$ using power=2:

$$
d=\left(\left(d_{\mathrm{Geo}}^{1.3}+d_{\mathrm{FWs} 50}^{1.3}\right)^{2 / 1.3}+d_{\mathrm{FWe} 50}^{2}\right)^{1 / 2},
$$

where the chromatic FW50 blur is estimated by $d_{\mathrm{FWc} 50}$ $=0.34 M\left(X_{A}^{*}(\Delta V / V) \alpha_{a}+T_{A}^{*} \beta_{0}(\Delta V / V)\right)$. The RPS spot size is $133 \mathrm{~nm}$ for the central beam and $138 \mathrm{~nm}$ for the outermost beam.

In principle, by adjusting diameters of outer lenses, the field curvature can be fully compensated. The relationship between the focal length and lens radius is shown in Fig. 8(a). The radius enlargement for outer lenses is calculated and plotted in Fig. 8(b). Distortion may be avoided by adjusting the position of microlenses. However, in practice, the field curvature and distortion compensation are limited by the lithography resolution and the accuracy of pattern transfer from lithography to dry etching. For the current settings, a $10 \mathrm{~nm}$ deviation from the ideal lens diameter gives a blur around $8 \mathrm{~nm}$, and the position error from microfabrication translates by a factor of 2 to probe position error.

\section{CONCLUSIONS}

The two-electrode immersion microlens array presents superior properties for focusing inclined beams when compared to the three-electrode Einzel lens. The total blur is minimized by offsetting the aperture. Other developments include integrating the aperture array with submicron alignment resolution, and correcting field curvature by adjusting diameters of outer lenses. These make the application in multibeam systems possible.
A 100-beam source unit is designed as an example. The design avoids angular errors due to spherical and chromatic aberration of the collimator lens in a traditional multibeam source. The multibeam source is capable of delivering a total current of $2.15 \mathrm{nA}$ at the wafer with uniform spot sizes.

In some cases, it is possible to use single-electrode microlenses, or aperture lenses. These turn out to have even smaller aberrations than two-electrode immersion lenses. An analysis of those systems can be found in Refs. 23 and 24 .

${ }^{1}$ P. Kruit, Microelectron. Eng. 84, 1027 (2007)

${ }^{2}$ L. P. Muray, C. S. Silver, and J. P. Spallas, J. Vac. Sci. Technol. B 24, 2945 (2006).

${ }^{3}$ J. P. Spallas, C. S. Silver, and L. P. Muray, J. Vac. Sci. Technol. B 24, 2892 (2006).

${ }^{4}$ T. Haraguchi, T. Sakazaki, T. Satoh, M. Nakano, S. Hamaguchi, T. Kiuchi, H. Yabara, and H. Yasuda, J. Vac. Sci. Technol. B 22, 985 (2004).

${ }^{5}$ E. Yin, A. D. Brodie, F. C. Tsai, G. X. Guo, and N. W. Parker, J. Vac. Sci. Technol. B 18, 3126 (2000).

${ }^{6}$ M. Mankos, S. Coyle, A. Fernandez, A. Sagle, P. Allen, W. Owens, J. Sullivan, and T. H. P. Change, J. Vac. Sci. Technol. B 18, 3010 (2000).

${ }^{7}$ L. R. Baylor et al., J. Vac. Sci. Technol. B 22, 3021 (2004).

${ }^{8}$ Osamu Kamimura et al., J. Vac. Sci. Technol. B 25, 140 (2007).

${ }^{9}$ H. Yasuda, S. Arai, J. Kai, Y. Ooae, T. Abe, S. Maruyama, and T. Kiuchi, J. Vac. Sci. Technol. B 14, 3813 (1996).

${ }^{10}$ M. Muraki and S. Gotoh, J. Vac. Sci. Technol. B 18, 3061 (2000).

${ }^{11}$ Stefan Eder-Kapl et al., Microelectron. Eng. 83, 968 (2006).

${ }^{12}$ M. J. van Bruggen, B. van Someren, and P. Kruit, J. Vac. Sci. Technol. B 23, 2833 (2005).

${ }^{13}$ S. Tanimoto et al., J. Vac. Sci. Technol. B 25, 380 (2007).

${ }^{14}$ P. Kruit, Publication No. US2007029509.

${ }^{15}$ Kenji Kurihara, J. Vac. Sci. Technol. B 4, 1251 (1986).

${ }^{16}$ B. Lencova and M. Lenc, Optik (Jena) 105, 121 (1997).

${ }^{17}$ B. Lencova and M. Lenc, Optik (Jena) 97, 121 (1994).

${ }^{18}$ B. Lencova and G. Wisselink, Electron Optical Design Program Packages ELD 3.7 (2002).

${ }^{19}$ J. E. Barth, and P. Kruit, Optik (Jena) 101, 101 (1996).

${ }^{20}$ V. K. Zworykin, G. A. Morton, E. G. Ramberg, J. Hillier, and A. W. Vance, Electron Optics and the Electron Microscope (Wiley \& Sons, New York, 1945).

${ }^{21}$ Jon Orloff, Handbook of Charged Particle Optics (CRC, Boca Raton, FL, 1997).

${ }^{22}$ A. K. Dokania, J. F. M. Velthuis, Yanxia Zhang, and Pieter Kruit, J. Vac. Sci. Technol. B 25, 504 (2007).

${ }^{23}$ Yanxia Zhang and P. Kruit, Presented in CPO7, [Nucl. Instrum. Methods Phys. Res. A (submitted)].

${ }^{24}$ Yanxia Zhang and P. Kruit, J. Vac. Sci. Technol. B 25, 2239 (2007). 